\title{
Percepción del liderazgo transformacional y gestión del talento humano en el personal administrativo de una Universidad Privada De Lima
}

Perception of transformational leadership and management of human talent in the administrative staff of a Private University of Lima

Percepção da liderança transformacional e gestão do talento humano na equipe administrativa de uma Universidade Privada de Lima

\section{ARTÍCULO ORIGINAL}

Milagritos Simona Barriga Vásquez

milabarriga@hotmail.com

https://orcid.org/0000-0002-4616-8058

Universidad San Martin de Porres
Claudia Virginia Cortez Chávez

claudiacortezch@gmail.com

Universidad Científica del Sur

Recibido 04 de Setiembre 2021 | Arbitrado y aceptado 08 de Setiembre 2021 | Publicado en 10 Setiembre 2021

\section{RESUMEN}

El presente artículo reporta sobre la relación entre las variables percepción del liderazgo transformacional y gestión del talento humano en el personal administrativo de una universidad privada de Lima. El estudio se ajustó al nivel de investigación descriptiva y al diseño descriptivo correlacional. La muestra fue obtenida por muestreo no probabilístico de tipo intencionado, quedando conformada por 44 trabajadores administrativos. Se administró el Cuestionario de Evaluación del Liderazgo Transformacional, así como la Escala de Gestión del Talento Humano. El análisis mostró que entre la percepción del liderazgo tranformacional y la gestión del talento humano se registra un valor de correlación $\mathrm{r}=0,752$, significativo al nivel de $\mathrm{p}<0,01$. Asimismo, se establecieron entre las cuatro dimensiones de la percepción del liderazgo transformacional y la gestión del talento humano, correlaciones significativas al nivel de $\mathrm{p}<0,01$, siendo la más alta la correspondiente a la dimensión: Reconocimiento laboral $(\mathrm{r}=0,650)$. Además, los hallazgos indican que la percepción del liderazgo transformacional y las dimensiones de gestión del talento humano correlacionan significativamente al nivel de $\mathrm{p}<0,01$, registrándose mayor correlación en la dimensión: Desarrollar personas $(r=0,685)$. En conclusión, existe relación significativa entre la percepción del liderazgo transformacional y la gestión del talento humano en el personal administrativo de la universidad considerada en el estudio.

Palabras Clave: Condiciones de trabajo, satisfacción laboral, enfermeros

\section{ABSTRACT}

This article reports on the relationship between the variables perception of transformational leadership and management of human talent in the administrative staff of a private university in Lima. The study was adjusted to the descriptive research level and the correlational descriptive design. The sample was obtained by intentional nonprobabilistic sampling, being made up of 44 administrative workers. The Transformational Leadership Assessment Questionnaire was administered, as well as the Human Talent Management Scale. The analysis showed that a correlation value $r=$ 0.752 is recorded between the perception of transformational leadership and the management of human talent, significant at the level of $p<0.01$. Likewise, among the four dimensions of the perception of transformational leadership and the management of human talent, significant correlations were established at the level of $p$ $<0.01$, the highest being the one corresponding to the dimension: Job recognition $(r=0.650)$. In addition, the findings indicate that the perception of transformational leadership and the dimensions of human talent management correlate significantly at the level of $p<0.01$, registering a higher correlation in the dimension: Developing people $(r=0.685)$. In conclusion, there is a significant relationship between the perception of transformational leadership and the management of human talent in the administrative staff of the university considered in the study.

Key Words: Working conditions, job satisfaction, nurses
\end{abstract}

\section{RESUMO}

Este artigo relata a relação entre as variáveis percepção da liderança transformacional e gestão do talento humano no corpo administrativo de uma universidade privada de Lima. O estudo foi ajustado ao nível de pesquisa descritiva e ao desenho descritivo correlacional. A amostra foi obtida por amostragem não probabilística intencional, sendo composta por 44 trabalhadores administrativos. O Questionário de Avaliação de Liderança Transformacional foi aplicado, bem como a Escala de Gestão de Talentos Humanos. A análise mostrou que se regista um valor de correlação $r=0,752$ entre a percepção da liderança transformacional e a gestão do talento humano, significativo ao nível de $\mathrm{p}$ $<0,01$. Da mesma forma, entre as quatro dimensões da percepção da liderança transformacional e da gestão do talento humano, foram estabelecidas correlações significativas ao nível de $\mathrm{p}<0,01$, sendo a maior a correspondente à dimensão: Reconhecimento do trabalho $(\mathrm{r}=0,650)$. Além disso, os achados indicam que as dimensões percepção da liderança transformacional e gestão do talento humano se correlacionam significativamente ao nível de $\mathrm{p}<0,01$, com maior correlação na dimensão: Pessoas em desenvolvimento $(r=0,685)$. Conclui-se que existe uma relação significativa entre a percepção da liderança transformacional e a gestão do talento humano no quadro administrativo da universidade considerada no estudo.

Palavras-chave: Condições de trabalho, satisfação no trabalho, enfermeiros 


\section{INTRODUCCIÓN}

La educación en el Perú y en muchos países de Latinoamérica se ha visto afectada por una serie de cambios tanto internos como externos, lo cual ha conllevado a reestructuraciones significativas y a una reorientación del sistema educativo, que exige un cambio de fondo y de forma, convirtiéndose así en un verdadero reto en la gestión de las instituciones educativas, particularmente el de la gerencia educativa, enfocando a las personas y sus relaciones, considerando qué se debe aprender, cómo trabajar en grupo, como intermediar en la solución de conflictos, etc.

Desde la visión socioeducativa, conocer en las organizaciones educativas aquellas actitudes y comportamientos limitantes expresados a través de la visión del liderazgo y el clima organizacional, permite preparar programas de apoyo formativo para la superación de las mismas. En relación al sistema educativo, los hallazgos permiten orientar a directores y a docentes en aspectos vinculados al estilo de liderazgo transformacional, así como a la gestión del talento humano, lo cual permite cumplir con la misión y la visión organizacional, optimizando la calidad educativa.

Para Ferreiro y Alcázar (2005), el líder es el que encabeza una organización familiar, empresarial, educativa, sin fines de lucro, etc., cuyos integrantes o trabajadores laboran siguiendo una misión trazada previamente; no obstante, la influencia que puede ejercer un líder en sus subordinados puede ser positiva o negativa en función a si se han cumplido o no con las expectativas laborales y de producción. Chiavenato (1993) refiere que el liderazgo viene a a ser el efecto que ejerce el directivo en su relación comunicacional con las personas de su entorno y con los empleados de una determinada organización; en tal sentido, líder es el que tiene la capacidad para conducir a un grupo de personas en base a lineamientos de planificación y manteniendo el respeto mutuo, encontrándose el liderazgo influido por diversos factores que pueden favorecer o ir en detrimento del trabajo que se realiza.

Específicamente, el liderazgo transformacional cobra un rol importante en las instituciones que se dedican al campo educativo, como las universidades, que tienen el reto de reformarse para responder a las necesidades sociales. Para Villalón (2014), el liderazgo transformacional es un liderazgo carismático, visionario, transformativo, más flexible e inclusivo, comunitario y democrático. Al respecto, Vázquez (2013) señala que “el líder transformacional rompe los esquemas organizacionales para crear una visión del 
futuro e invierte mucho tiempo en compartirla. A través de este proceso, el líder aclara el presente y muestra cómo el pasado lo ha influenciado, para finalmente utilizar a ambos para transformar el futuro" (p. 78).

Considerando la tipología del liderazgo transformacional, Burns (1978) distingue tres tipos de líderes transformacionales en el contexto social, aunque no los reconoce como categorías; estos tipos tienen las características transformacionales, pero destacan en algún medio específico; estos son: Liderazgo intelectual, que trabaja con ideas normativas y analíticas, no está desvinculado de su contexto social, sino que intenta cambiarlo, y tiene una conciencia de propósito; liderazgo reformador, que cuenta con habilidades políticas excepcionales, debe ser estratega y proclive a las alianzas, y es un liderazgo moral, con gran poder de negociación; liderazgo revolucionario, que encabeza una transformación de todo un sistema social, es un líder totalmente dedicado a la causa, que puede llegar hasta ser mártir, genera una conciencia social y política entre los líderes y los seguidores.

Uno de los factores que influyen en el liderazgo transformacional hace referencia, desde luego, a la denominada cultura organizacional. Se puede afirmar, entonces, que el tipo de cultura de una organización determina, en cierta medida, la presencia de un liderazgo transformacional. Sin embargo, también el liderazgo transformacional puede afectar el desarrollo de la cultural organizacional; es decir, la relación entre ambos conceptos es recíproca (Bass y Avolio, 1993). Para tal hecho, es importante considerar que los líderes transformacionales influencian tres áreas de la cultura organizacional: La mentalidad de la gente de la organización, la cultura entre la gente de la misma, y la cultura más allá de la gente de la institución. Si se concibe en un plano más amplio la cultura organizacional, es necesario analizar otros aspectos: Artefactos visibles, que corresponde a la arquitectura de la organización, su tecnología, la disposición de oficinas, la forma de vestir de las personas, las formas de hablar y comportarse y los documentos públicos; valores que gobiernan el comportamiento, que es el siguiente nivel de profundidad, y son difíciles de observar directamente, por lo que deben inferirse a través de entrevistas o del análisis de contenido de documentos; y supuestos subyacentes, que son inconscientes y sobre los cuales verdaderamente se construye la cultura organizacional, son poderosos porque no son debatibles, dada su condición inconsciente (Bass y Avolio, 1993). 
La necesidad de cambios en gestión que se experimentan en el mundo de hoy, hace que se requiera de la creación de un nuevo sistema organizacional y su institucionalización a través de nuevas formas de administración. Brown y Eisenhardt (1997) afirman que se requiere de líderes exitosos que sean: Proveedores de responsabilidades y prioridades claras, en un marco de comunicación constante y libertad para la improvisación, que creen una estructura con muy pocas reglas que promueva la creatividad de sus seguidores; exploradores del futuro mediante la experimentación con una gran variedad de pruebas de bajo costo, que permita el aprendizaje organizacional, fundamental para enfrentar un futuro incierto; vinculadores del presente con el futuro, a través de los proyectos presentes y procedimientos transicionales que poco a poco se van haciendo familiares en la organización y que permiten el cambio cuando se ha creado un ritmo en ella. De esa manera, el líder transformacional provee de cambio y movimiento a la organización; por ello, debe tener una clara visión de las metas a alcanzar para guiar a la institución en nuevas direcciones. Este tipo de líder enfatiza las nuevas posibilidades y promueve una visión de futuro porque es guiado por un fuerte sentido de propósito. El líder transformacional cuestiona todo y promueve un estilo de pensamiento no tradicional. Asimismo, puede cambiar los valores básicos, creencias y actitudes de sus seguidores, mediante la articulación de una visión, el logro de su aceptación en los diferentes grupos de la organización y la provisión del apoyo individual que les motiva a alcanzarla.

Algunas cualidades del líder transformacional lo hacen apto para liderar ciertos tipos de cambios. Bass y Riggio (2006) sostienen que el liderazgo transformacional es mejor para las situaciones no rutinarias. Por su lado, Leithwood y Steinbach (1993) afirman que el pensamiento experto es particularmente crucial para los líderes, porque les provee de la flexibilidad cognitiva que requieren para sus contextos tan cambiantes. Este tipo de pensamiento crea una propensión a la actuación transformacional. El líder debe enmarcar el proceso de cambio en la necesidad de crecimiento personal de los seguidores, que sólo así podrán verlo atractivo. El proceso también puede ser facilitado si muestra consideración individual y provee apoyo y guía al personal involucrado. De esta forma, el líder transformacional será un gran facilitador del proceso si promueve una cultura que privilegie la toma de decisiones grupales.

Una variable interesante, que guarda afinidad con una de las afirmaciones de Brown y Eisenhardt (1997), respecto a que se requieren líderes exitosos que generen cambio y promuevan la creatividad en sus seguidores, es la correspondiente a la gestión del talento 
humano. Según la Real Academia Española de la Lengua, este concepto hace mención a la persona inteligente con capacidad de entender y con aptitudes que le permiten desempeñarse en una ocupación determinada. Por su parte, Jericó (2001) señala que el talento requiere capacidades juntamente con compromiso y acción, los tres al mismo tiempo; es decir, si se da el caso que el trabajador dispone de capacidades y actúa en el momento, pero no se compromete con el proyecto, puede que alcance resultados., pero su falta de motivación le impedirá innovar o proponer cosas más allá de las impuestas por su jefe. Si ocurre que el profesional tiene capacidades y compromiso, pero cuando actúa ya ha pasado el momento, tampoco obtendrá los resultados deseados por la simple razón de que alguno se le ha podido adelantar.

Concerniente a la definición de talento humano, García et al. (2008) afirman que se trata de una actividad estratégica de apoyo y soporte a la dirección, compuesta por un conjunto de políticas, planes, programas y actividades, con el objeto de obtener, formar, motivar, retribuir y desarrollar al personal requerido para generar y potencializar el management, la cultura organizacional y el capital social, donde se equilibran los diferentes intereses que convergen en la organización para lograr los objetivos de manera efectiva. Chiavenato (2009) lo define como "el conjunto de políticas y prácticas necesarias para dirigir los aspectos administrativos en cuanto a las "personas" o los recursos humanos, como el reclutamiento, la selección. La formación, las remuneraciones y la evaluación del desempeño" (p. 9). Cuesta (2010), desde el punto de vista estratégico, lo define como "el conjunto de decisiones y acciones directivas en el ámbito organizacional que influyen en las personas, buscando el mejoramiento continuo, durante la planeación, implantación y control de las estrategias organizacionales, considerando las interacciones con el entorno" (p. 3). Y, por último, para Ibáñez (2011) viene a ser "el proceso administrativo aplicado al acrecentamiento y conservación del esfuerzo humano; las experiencias, la salud, los conocimientos, las habilidades y todas las cualidades que posee el ser humano como miembro de la organización, en beneficio de la propia organización y del país en general” (p. 17).

En cuanto a las funciones de la gestión del talento, Chiavenato (2009) refiere que la gestión del talento humano está constituida por las personas y las organizaciones. Las personas, como trabajadores permanecen gran parte de sus vidas en las empresas, las que dependen del individuo para funcionar y alcanzar el éxito. Es de conocimiento que el trabajo consume el tiempo considerable de la vida y del esfuerzo de las personas que 
dependen de él para de alguna manera vivir y alcanzar el éxito personal; es así que hacer un desglose del trabajo de la existencia de las personas es muy difícil, casi imposible, por la importancia y el efecto que tiene en ellas. Por tal razón, Chiavenato (2009) concreta la gestión del talento humano como un área bastante sensible a la manera de pensar que predomina en las organizaciones. Es por ello, que para gestionar el talento humano dentro de las empresas, impera la importancia de tener presente ciertos elementos, que hacen posible esta ardua labor, tanto para su ejecución como para llevar un control sobre ellos. Estos elementos, que son nueve, son: Planificación de necesidades de personal; análisis y descripción de puestos de trabajo; administración de compensaciones; capacitación y desarrollo; plan de desarrollo de carrera profesional; evaluación del desempeño; comunicación interna; motivación laboral; y dirección de equipos de trabajo.

En el presente estudio se consideran dos temas muy importantes: el liderazgo transformacional y la gestión del talento humano, aspectos que son relevantes en las universidades, donde los docentes y trabajadores en general tienen que mostrar una actitud de compromiso, de solidaridad, honestidad, y en donde el profesionalismo y las habilidades sociales deben ponerse a flote para el logro de los objetivos y misión de cada institución. Según Gibson et al. (2001), elevar el rendimiento y la productividad del capital humano sigue siendo un reto, mientras que para los directivos contar con colaboradores que tengan mística, espíritu de cuerpo o la «camiseta bien puesta» (que se propongan como meta el mejoramiento de la productividad), más que una aspiración, es una meta que se debe lograr. Es decir, directivos y trabajadores que entiendan que la única manera de alcanzar el progreso y el bienestar individual y de la organización es elevando el rendimiento y la productividad, y ello requiere de trabajadores comprometidos con su organización. En resumen, el progreso tanto individual como organizacional está circunscrito al desarrollo de la productividad, y esta depende en gran medida del compromiso y mística que tengan las personas que forman parte de la organización.

\section{OBJETIVOS DE LA INVESTIGACIÓN}

\section{Objetivo general}

Determinar la relación entre la percepción del liderazgo transformacional y la gestión del talento humano en el personal administrativo de una universidad privada de Lima. 


\section{Objetivos específicos}

Establecer la relación entre la percepción del liderazgo transformacional y las dimensiones de la gestión del talento humano en el personal administrativo de una universidad privada de Lima.

Establecer la relación entre las dimensiones de la percepción del liderazgo transformacional y la gestión del talento humano en el personal administrativo de una universidad privada de Lima.

\section{MÉTODO}

\section{Nivel y diseño}

Este estudio se ajustó al nivel de investigación descriptiva y al diseño descriptivo correlacional (Sánchez y Reyes, 2015).

\section{Participantes}

La población estuvo integrada por 91 trabajadores administrativos que laboran en una universidad privada de Lima Metropolitana.

La muestra quedó constituida por 44 trabajadores administrativos, la cual se seleccionó mediante muestreo no probabilístico de tipo intencionado.

\section{Instrumentos}

\section{Cuestionario de Evaluación del Liderazgo Transformacional}

La percepción del liderazgo transformacional y sus dimensiones se han medido utilizando el Cuestionario de Evaluación del Liderazgo Transformacional. Este instrumento consta de 25 ítems con opciones de respuesta elaboradas según técnica de Likert, específicamente de tipo frecuentista, por lo que las alternativas planteadas son: Nunca (1), Casi Nunca (2), A veces (3), Casi siempre (4) y Siempre (5). Los ítems están agrupados en cuatro dimensiones, tales como: Compromiso prospectivo (ítems 1-6), Reconocimiento laboral (ítems 7-12), Responsabilidad funcional (ítems 13-18) y Motivación intelectual (ítems 19-25). Cabe señalar que, para efectos de la calificación, se contabilizan los puntajes de las respuestas a los ítems de cada dimensión, considerando para el total de la escala la sumatoria de los puntajes de las dimensiones. La única 
salvedad, al momento de calificar, es tener en cuenta que el ítem 18 es negativo, es decir, se califica en dirección inversa.

En cuanto a la validez del cuestionario, se aplicó el método de juicio de expertos, para lo cual se contó con la participación de tres especialistas en el tema, quienes otorgaron valoraciones equivalentes al 90\%, estimándose, de esa manera, un alto grado de concordancia que supera el mínimo establecido por Guilford (1954); en consecuencia, se puede afirmar que el instrumento es válido para su administración a la muestra de estudio.

Respecto a la confiabilidad, según los datos de 50 docentes reportados por Suarez y Cabrejos (2019), se registran los siguientes valores obtenidos del coeficiente alfa de Cronbach para las dimensiones y escala total del cuestionario: Compromiso prospectivo $($ alfa $=0,614)$, Reconocimiento laboral $($ alfa $=0,593)$, Responsabilidad funcional $($ alfa $=$ $0,719)$, Motivación intelectual $($ alfa $=0,560)$ y escala total $(0,882)$. Es de resaltar que Suarez y Cabrejos (2019) hicieron uso de una versión abreviada que consta de 12 ítems en total, conformándose para cada dimensión solo tres reactivos. Posiblemente los bajos valores alfa registrados se vean reflejados en esta conformación de un reducido número de ítems por cada dimensión.

Del mismo modo, los resultados de confiabilidad del instrumento calculados para este estudio fueron: Compromiso prospectivo (alfa $=0,889$ ), Reconocimiento laboral $($ alfa $=0,743)$, Responsabilidad funcional $($ alfa $=0,673)$, Motivación intelectual $($ alfa $=$ 0,787) y escala total (alfa $=0,878)$. En conclusión, de acuerdo a estos resultados, se obtuvieron valores que indican una consistencia interna aceptable, por lo cual la aplicación del cuestionario en mención a la muestra en estudio resulta suficientemente adecuada.

\section{Escala de Gestión del Talento Humano}

Este instrumento fue elaborado por Soto (2017), y consta de 36 ítems con opciones de respuesta de tipo frecuentista, etiquetadas como: Nunca (1), Casi Nunca (2), A veces (3), Casi siempre (4) y Siempre (5). Las dimensiones que mide son seis; sin embargo, para los fines de este estudio, las autoras consideraron una versión con solo cuatro de las seis dimensiones que incluye la escala original. De esta manera, se incluyeron los ítems 
correspondientes a: Recompensar personas (ítems 1-7), Desarrollar personas (ítems 814), Retener personas (ítems 15-21) y Supervisar personas (ítems 22-27).

Con referencia a la validez de la Escala, se aplicó, como en el caso del instrumento anterior, el método de juicio de expertos, cuyos participantes asignaron valoraciones equivalentes al 90\%, estableciéndose, de ese modo, un alto grado de concordancia, pues el porcentaje consensuado es mucho mayor al límite de $80 \%$ indicado por Guilford (1954); por lo tanto, se confirma que el instrumento es válido para su aplicación a la muestra seleccionada.

Concerniente a la confiabilidad, en base a los datos de 105 trabajadores administrativos de una UGEL de Lima Metropolitana, registrados por Soto (2017) en su trabajo de investigación, se obtuvieron los siguientes valores alfa de Cronbach para las dimensiones y escala total de la Escala de Gestión del Talento Humano: Recompensar personas $($ alfa $=0,791)$, Desarrollar personas $($ alfa $=0,749)$, Retener personas $($ alfa $=$ 0,757), Supervisar personas (alfa $=0,763)$ y escala total $(0,929)$.

De otro lado, las autoras de este estudio, en la obtención de la confiabilidad del instrumento, encontraron similares valores alfa de Cronbach, tales como: Recompensar personas $($ alfa $=0,703)$, Desarrollar personas $($ alfa $=0,809)$, Retener personas $($ alfa $=$ $0,710)$, Supervisar personas $(\mathrm{alfa}=0,786)$ y escala total $(0,889)$. En consecuencia, estos resultados permiten concluir y confirmar que la Escala mantiene en los ítems de cada una de sus dimensiones, una consistencia interna aceptable, mientras que a escala global presenta un alto niveles de consistencia interna, en virtud de lo cual califica como un instrumento confiable e idóneo para su administración a la muestra de investigación.

\section{RESULTADOS}

\section{Percepción del liderazgo transformacional}

Se observa en la tabla 1 , que el 54,5\% de los trabajadores administrativos encuestados presentan una percepción en nivel regular del liderazgo transformacional. Asimismo, teniendo en cuenta cada una de las dimensiones, se aprecia, en cuanto a Compromiso prospectivo, que el $72,7 \%$ del personal administrativo lo percibe en nivel alto. También un 72,7\% percibe la dimensión: Reconocimiento laboral, pero en nivel regular. Del mismo modo, el 54,5\% tiene una percepción regular acerca de la dimensión: 
Responsabilidad funcional. Similar porcentaje se registra concerniente al nivel regular de la dimensión: Motivación intelectual.

\section{Tabla 1}

Resultados descriptivos en frecuencias y porcentajes sobre la percepción del liderazgo transformacional y sus dimensiones

\begin{tabular}{lcrrrr}
\hline Variable / Dimensión & Estadístico & & Nivel & & Total \\
& & Alto & Regular & Bajo & \\
\hline $\begin{array}{l}\text { Percepción del liderazgo } \\
\text { transformacional }\end{array}$ & $\mathrm{F}$ & 20 & 24 & 0 & 44 \\
\hline $\begin{array}{l}\text { Compromiso } \\
\text { prospectivo }\end{array}$ & $\%$ & 45,5 & $\mathbf{5 4 , 5}$ & 0,0 & 100,0 \\
\hline Reconocimiento laboral & $\mathrm{F}$ & 32 & 12 & 0 & 44 \\
& $\%$ & $\mathbf{7 2 , 7}$ & 27,3 & 0,0 & 100,0 \\
\hline $\begin{array}{l}\text { Responsabilidad } \\
\text { funcional }\end{array}$ & $\mathrm{F}$ & 4 & 32 & 8 & 44 \\
\hline Motivación intelectual & $\%$ & 45,5 & $\mathbf{5 4 , 5}$ & 0,0 & 100,0 \\
\hline & $\mathrm{F}$ & 20 & 24 & 0 & 44 \\
\hline
\end{tabular}

\section{Gestión del talento humano}

Se advierte en la tabla 2 , que, de acuerdo a lo respondido por el $52,3 \%$ de los trabajadores administrativos, la gestión del talento humano se estima en un nivel eficaz. Correspondiente a la dimensión: Recompensar personas, ésta se ubica en un nivel medianamente eficaz, con el 72,7\% del personal administrativo evaluado. Según lo contestado por el 52,3\%, la dimensión: Desarrollar personas se encuentra en un nivel eficaz. También la dimensión: Retener personas se mantiene en un nivel eficaz, donde se agrupa el 86,4\% de los encuestados. Y relativo a la dimensión: Supervisar personas, ésta se coloca en un nivel medianamente eficaz, con el 59,1\% de los examinados. 


\section{Tabla 2}

Resultados descriptivos en frecuencias y porcentajes sobre la gestión del talento humano y sus dimensiones

\begin{tabular}{|c|c|c|c|c|c|}
\hline \multirow[b]{2}{*}{ Variable / Dimensión } & \multirow[b]{2}{*}{ Estadístico } & \multicolumn{3}{|c|}{ Nivel } & \multirow[b]{2}{*}{ Total } \\
\hline & & Eficaz & $\begin{array}{l}\text { Medianamente } \\
\text { eficaz }\end{array}$ & Ineficaz & \\
\hline \multirow{2}{*}{$\begin{array}{l}\text { Gestión del talento } \\
\text { humano }\end{array}$} & $\mathrm{F}$ & 23 & 20 & 1 & 44 \\
\hline & $\%$ & 52,3 & 45,5 & 2,3 & 100,0 \\
\hline \multirow{2}{*}{ Recompensar personas } & $\mathrm{F}$ & 10 & 32 & 2 & 44 \\
\hline & $\%$ & 22,7 & 72,7 & 4,5 & 100,0 \\
\hline \multirow{2}{*}{ Desarrollar personas } & $\mathrm{F}$ & 23 & 18 & 3 & 44 \\
\hline & $\%$ & 52,3 & 40,9 & 6,8 & 100,0 \\
\hline \multirow{2}{*}{ Retener personas } & $\mathrm{F}$ & 38 & 6 & 0 & 44 \\
\hline & $\%$ & 86,4 & 13,6 & 0,0 & 100,0 \\
\hline \multirow{2}{*}{ Supervisar personas } & $\mathrm{F}$ & 15 & 26 & 3 & 44 \\
\hline & $\%$ & 34,1 & 59,1 & 6,8 & 100,0 \\
\hline
\end{tabular}

\section{Prueba de normalidad de datos}

Para proceder con la prueba de hipótesis, primero se determinó si las dimensiones y variables involucradas en el tratamiento de análisis inferencial presentaban o no distribución normal en sus puntuaciones. De manera que se optó por aplicar la prueba de normalidad de datos de Shairo-Wilk, adecuada para muestras menores a 50 sujetos. Los resultados obtenidos se registran en la tabla 3 , donde se aprecia que los valores de significación de cada una de las dimensiones y escala total de las variables de estudio Liderazgo transformacional y Gestión del talento humano permiten aceptar la hipótesis nula que establece normalidad de datos. En vista de estos hallazgos, se eligió como prueba paramétrica la de correlación lineal de Pearson, para su aplicación a las hipótesis de investigación. 


\section{Tabla 3}

Normalidad de datos de las variables y dimensiones de estudio

\begin{tabular}{lccc}
\hline Variable / Dimensión & Estadístico & gl & Significación \\
\hline Liderazgo transformacional &, 987 & 44 &, 903 \\
Compromiso prospectivo &, 962 & 44 &, 150 \\
Reconocimiento laboral &, 977 & 44 &, 500 \\
Responsabilidad funcional &, 973 & 44 &, 394 \\
Motivación intelectual &, 984 & 44 &, 785 \\
\hline Gestión del talento humano &, 981 & 44 &, 692 \\
Recompensar personas &, 980 & 44 &, 645 \\
Desarrollar personas &, 962 & 44 &, 157 \\
Retener personas &, 954 & 44 &, 079 \\
Supervisar personas &, 974 & 44 &, 407 \\
\hline
\end{tabular}

Relación entre percepción del liderazgo transformacional y gestión del talento humano Para la correlación entre las variables de estudio, se obtuvo un coeficiente de Pearson $(\mathrm{r}=0,752)$ estadísticamente significativo al nivel de $\mathrm{p}<0,01$ (tabla 4), resultado que permite inferir que entre ambas variables existe una relación significativa, con magnitud cercana a la correlación fuerte, según la clasificación propuesta por Elorza (2008). Cabe señalar que la relación se establece, además, como positiva, pues en la medida que se incrementa la puntuación en percepción del liderazgo transformacional, también se eleva la puntuación en gestión del talento humano. Por consiguiente, se comprueba la hipótesis general de estudio.

\section{Tabla 4}

Resultados de correlación de Pearson entre las variables Percepción del liderazgo transformacional y Gestión del talento humano.

\begin{tabular}{llc}
\hline & & Gestión del talento humano \\
\hline & Correlación de Pearson & $0,752 * *$ \\
Percepción del liderazgo & Sig. (unilateral) & 0,000 \\
transformacional & $\mathrm{N}$ & 44 \\
\hline$* *$ Significativo al nivel de $\mathrm{p}<0,01$. &
\end{tabular}


Relación entre la percepción del liderazgo transformacional y las dimensiones de gestión del talento humano

Al correlacionar percepción del liderazgo transformacional con cada una de las dimensiones de gestión del talento humano, se encontró que todos los coeficientes de Pearson obtenidos son estadísticamente significativos al nivel de $\mathrm{p}<0,01$ (tabla 5). Estos registros evidencian una relación significativa con correlaciones moderadas en todos los casos (Elorza, 2008). También las correlaciones establecidas son positivas, pues mientras se eleva la puntuación en percepción del liderazgo transformacional, tiende a subir la puntuación en cada una de las dimensiones de gestión del talento humano. Por consiguiente, se comprueba la primera hipótesis específica de estudio.

\section{Tabla 5}

Resultados de correlación de Pearson entre la variable Percepción del liderazgo transformacional y las dimensiones de Gestión del talento humano $(\mathrm{n}=44)$

\begin{tabular}{llcccc}
\hline & & $\begin{array}{l}\text { Recompensar } \\
\text { personas }\end{array}$ & $\begin{array}{l}\text { Desarrollar } \\
\text { personas }\end{array}$ & $\begin{array}{l}\text { Retener } \\
\text { personas }\end{array}$ & $\begin{array}{l}\text { Supervisar } \\
\text { personas }\end{array}$ \\
\hline $\begin{array}{l}\text { Percepción del } \\
\text { liderazgo }\end{array}$ & $\begin{array}{l}\text { Correlación de } \\
\text { Pearson }\end{array}$ & $0,589^{* *}$ & $0,685^{* *}$ & $0,524^{* *}$ & $0,565^{* *}$ \\
transformacional & Sig. (unilateral) & 0,000 & 0,000 & 0,000 & 0,000 \\
\hline
\end{tabular}

** Significativo al nivel de $\mathrm{p}<0,01$.

\section{Relación entre las dimensiones del liderazgo transformacional y la gestión del}

\section{talento humano}

Luego de calcular las correlaciones en las dimensiones de percepción de liderazgo transformacional con gestión del talento humano, se obtuvieron coeficientes de Pearson estadísticamente significativos al nivel de $\mathrm{p}<0,01$ (tabla 6). Estos valores remiten a una relación significativa con correlación débil respecto a la dimensión: Compromiso prospectivo $(\mathrm{r}=0,457)$, pero con correlaciones moderadas en las otras dimensiones (Elorza, 2008). También las correlaciones establecidas son positivas, pues mientras se eleva la puntuación en percepción del liderazgo transformacional, tiende a subir la puntuación en cada una de las dimensiones de gestión del talento humano. Por consiguiente, se comprueba la segunda hipótesis específica de estudio. 


\section{Tabla 6}

Resultados de correlación de Pearson entre las dimensiones de Percepción del liderazgo transformacional y la variable Gestión del talento humano $(n=44)$

\begin{tabular}{llc}
\hline & & Gestión del talento humano \\
\hline $\begin{array}{l}\text { Compromiso } \\
\text { prospectivo }\end{array}$ & Correlación de Pearson & $0,457^{* *}$ \\
\hline \multirow{2}{*}{ Reconocimiento laboral } & Sig. (unilateral) & 0,001 \\
& Correlación de Pearson & $0,650^{* *}$ \\
\hline \multirow{2}{*}{$\begin{array}{l}\text { Responsabilidad } \\
\text { funcional }\end{array}$} & Correlación de Pearson & 0,000 \\
\hline \multirow{2}{*}{ Motivación intelectual } & Sig. (unilateral) & $0,544^{* *}$ \\
& Correlación de Pearson & 0,000 \\
\hline
\end{tabular}

** Significativo al nivel de $\mathrm{p}<0,01$.

\section{DISCUSIÓN}

Los resultados que se han obtenido con el presente estudio permiten establecer, en primer lugar, que la correlación entre la percepción del liderazgo transformacional y la gestión del talento humano es significativa. Algunos estudios en nuestro medio corroboran, en cierta forma, este hallazgo. Al respecto, Chiuche (2016) encontró que el liderazgo transformacional se relaciona significativamente con la gestión del talento humano en un grupo de docentes de instituciones educativas de San Juan de Lurigancho. Por su parte, Santander et al. (2018) observaron en el personal de un grupo militar, que algunas dimensiones del liderazgo, tales como Estimulación intelectual $\mathrm{y}$ Consideraciones individualizadas, correlacionaban de manera significativa con las cuatro dimensiones de la gestión del talento humano (Recompensar, Desarrollar, Retener y Supervisar personas); es decir, demostraron, aunque parcialmente, la relación entre ambas variables. En un estudio similar desarrollado por Zeña (2016), se consigna que la gestión del potencial humano se relaciona directa y significativamente con el liderazgo pedagógico percibido por docentes de una institución educativa de Lima. Como señala Torres (2019), “el líder que se considera realmente competitivo en el mercado y empático dentro de la organización, adopta como estrategia de gestión del talento humano, ser un líder transformacional, aquel que entiende las necesidades de sus colaboradores, que se 
alía con ellos en pro de un beneficio mutuo, aquel que trabaja mancomunadamente con su equipo, y que con el fin de mantenerse a la vanguardia de los cambios acelerados y vertiginosos que da el mundo en la actualidad, les permite que realicen aportes creativos e innovadores..." (p. 16).

En segundo lugar, las evidencias muestran que la percepción del liderazgo transformacional correlaciona significativamente con las dimensiones de la gestión del talento humano (Recompensar, Desarrollar, Retener y Supervisar personas); asimismo, se registraron correlaciones significativas entre las dimensiones del liderazgo transformacional (Compromiso prospectivo, Reconocimiento laboral, Responsabilidad funcional y Motivación intelectual) y la gestión del talento humano. Estos resultados confirman la estrecha relación entre las dimensiones de ambas variables, dejando entrever, como refiere Vásquez (2013), un aporte importante del liderazgo transformacional a las organizaciones, en las cuales sus objetivos se centran en promover la creatividad y la innovación entre sus trabajadores para alcanzar mayor productividad y competitividad.

Algunos autores teorizan sobre la influencia del liderazgo transformacional en la gestión de determinados elementos relacionados con el talento humano; es el caso de Vidal (2017), que concluye que “...la correcta gestión del talento humano [...] proporciona equipos de trabajo motivados, alineados a la visión organizacional, acercándolos más a la consecución de los objetivos que sienten como suyos, por lo cual entregarán más de lo esperado para alcanzarlos” (p. 21). Esta autora sostiene, además, que el liderazgo transformacional está fuertemente relacionado con la gestión de equipos de trabajo, pues permite optimizar aquellos elementos esenciales para el éxito de un equipo de trabajo, siendo el fundamento de tal relación la orientación del liderazgo transformacional hacia el capital humano. Otra autora que buscó establecer una relación entre el liderazgo transformacional y el desarrollo de equipos de alto rendimiento, es Pico (2018), quien analizó las características y dinámica del liderazgo transformacional en el desarrollo de los aspectos que permiten gestionar el cambio organizacional mediante la sinergia de esfuerzos realizados por el equipo. Así también, Nader y Castro (2007) afirman que este equipo o grupo de personas que se desenvuelven en la organización necesita la conducción de parte de un líder, requieren su orientación, su seguimiento, así como una permanente motivación para el logro de los objetivos planeados, por lo que es 
necesario considerar la importancia que tiene un líder que lleve la batuta y dirija el concierto a buen término.

Finalmente, cabe mencionar el trabajo de Carvajal et al. (2020), quienes investigaron en empleados de la empresa Taco Bell de qué manera el liderazgo transformacional podía influir en la gestión del cambio en sus principales sedes. Los resultados obtenidos hicieron evidente la importancia del liderazgo transformacional en el logro de motivar a las personas a realizar sus labores, en concordancia con las metas de la organización. Señalaron, asimismo, que el liderazgo transformacional en la empresa se ajustó al modelo de cambio planteado por Kurt Lewin (1947, citado en Carvajal et al., 2020), en la medida que sugiere contar con personas influyentes dentro de la organización, que no sean las mismas que cuentan con el poder jerárquico, sino que sea alguien que cuente con las destrezas, habilidades y capacidades para dirigirse de forma asertiva a su equipo de trabajo y, de esta manera, cuidar porque se ejecuten las tres fases del modelo para aspirar a un cambio organizacional.

Estos son los resultados registrados en el estudio, que no son definitivos, ya que existen ciertas limitaciones al querer utilizarlos como marco referencial a determinadas explicaciones; son, en todo caso, aproximaciones relacionadas con el valor significativo que tiene la influencia de la percepción del liderazgo transformacional en la gestión del talento humano, en el contexto del sistema organizacional universitario.

\section{REFERENCIAS BIBLIOGRÁFICAS}

Bass B. y Avolio B. (1993). Transformational leadership and organizational culture. Public Administration Quarterly, 17(1), 112-118.

Bass, B. M. \& Riggio, R. (2006). Transformational leadership. Lawrence Erlbaum Associates Publishers.

Brown, S. L. \& Eisenhardt, K. M. (1997). The art of continuous change: Linking complexity theory and time-paced evolution in relentlessly shifting organizations. Administrative science quarterly, 42(1), 1-34. https://doi.org/10.2307/2393807

Burns, J. (1978). Leadership. Harper \& Row. 
Carvajal, I. Y., Peña, M. D., y Peña, K. A. (2020). Liderazgo transformacional en la gestión del cambio en la cadena de restaurantes Taco Bell [Seminario de profundización en organizacional, Universidad Cooperativa de Colombia sede Villavicencio].

https://repository.ucc.edu.co/bitstream/20.500.12494/17942/1/2020_liderazgo_transfor macional.pdf

Chiavenato, I. (2009). Gestión del talento humano. McGraw-Hill.

Chiavenato, I. (1993). Introducción a la teoría general de la administración. McGraw-Hill.

Chiuche, C. A. (2018). Liderazgo transformacional y la gestión del talento humano en docentes de la Red 06, UGEL 05, San Juan de Lurigancho. 2015 [Tesis de maestría, Universidad César Vallejo]. https://repositorio.ucv.edu.pe/bitstream/handle/20.500.12692/7872/Chiuche_CCA.pdf?s equence $=1 \&$ is Allowed $=\mathrm{y}$

Cuesta, A. (2010). Gestión del talento humano y del conocimiento. Ecoediciones.

Elorza, H. (2008). Estadística para las ciencias sociales, del comportamiento y de la salud. Cengage Learning.

Ferreiro, P y Alcázar, M. (2005). Gobierno de personas en la empresa. PADEscuela de Dirección de la Universidad de Piura.

García, M., Sánchez, K., y Zapata, Á. (2008). Perspectivas teóricas para el estudio de la gestión humana. Universidad del Valle.

Gibson, J., Ivancevich, J., y Donelly, J. (2001). Las organizaciones: comportamiento, estructura, procesos. McGraw-Hill / Interamericana.

Guilford, J. P. (1954). Psychometric methods. McGraw-Hill.

Ibáñez, M. (2011). Gestión del talento humano en la empresa. San Marcos. Jericó, P. (2001). Gestión del talento. Prentice Hall / Pearson Educación. 
Leithwood, K. y Steinbach, R. (1993). Liderazgo de calidad total: pensamientode expertos y práctica de transformación. Journal of Personnel Evaluation in Education, 7(4), 311- 338 .

Nader, M. y Castro, A. (2007). Influencia de los valores sobre los estilos de liderazgo: un análisis según el modelo de liderazgo transformacional-transaccional de $\begin{array}{lll}\text { Bass. } & \text { Universitas } & \text { 6(3), }\end{array}$ https://www.redalyc.org/pdf/647/64760318.pdf

Pico, L. M. (2018). Relación entre el liderazgo transformacional y el desarrollo de equipos de alto rendimiento. INNOVA Research Journal, 3(12), 28-37. https://dialnet.unirioja.es/descarga/articulo/6828550.pdf

Sánchez, H. y Reyes, C. (2015). Metodología y diseños en la investigación científica. Business Support Aneth.

Santander, M. D., Orrego, J. C., y Usquiano, S. L. (2018). Liderazgo transformacional y gestión del talento humano en el personal del Ejército del Perú de los contingentes en Misiones de Paz en la República de Haití [Tesis de maestría, Escuela Superior de Guerra del Ejército]. http://repositorio.esge.edu.pe/bitstream/handle/ESGEEPG/141/LIDERAZGO\%20TRA NSFORMACIONAL\%20Y\%20GESTI\%c3\%93N\%20DEL\%20TALENTO\%20HUMA NO.pdf?sequence $=1 \&$ is Allowed $=\mathrm{y}$

Soto, J. S. (2017). Gestión del talento humano y motivación laboral según personal de la Unidad de Gestión Educativa Local 04, Lima 2016 [Tesis de maestría, Universidad César Vallejo]. https://repositorio.ucv.edu.pe/bitstream/handle/20.500.12692/7189/Soto_SJS.pdf?seque $\underline{\text { nce }}=1 \&$ isAllowed $=y$.

Suarez, E. L. y Cabrejos, R. (2019). Liderazgo transformacional y productividad en los docentes de la escuela fiscal Antonio José de Sucre de la provincia de Guayas Ecuador. Ciencia y Desarrollo, 22(3), 37-47. http://dx.doi.org/10.21503/cyd.v22i3.1792

Torres, J. A. (2019). El liderazgo transformacional: estrategia para la gestión del talento humano enfocado a la aceptación del cambio organizacional [Ensayo de grado, 
Universidad

Militar

Nueva

Granada].

https://repository.unimilitar.edu.co/bitstream/handle/10654/21159/TorresRojasJulieAnd rea2019.pdf? sequence $=2 \&$ is Allowed $=\mathrm{y}$

Vázquez, A.(2013). Interdependencia entre e liderazgo transformacional, cultura organizacional y cambio educativo: una reflexión. REICE. Revista Iberoamericana sobre Calidad, Eficacia y Cambio en Educación, 11(1), 73-91. http://www.rinace.net/reice/numeros/arts/vol11num1/art5.pdf

Vidal, C. A. (2017). El liderazgo transformacional como herramienta en la gestión de equipos de trabajo [Ensayo de grado, Universidad Militar Nueva Granada]. https://repository.unimilitar.edu.co/bitstream/handle/10654/16256/VidalCruzCarolAndr ea2017.pdf? sequence $=1 \&$ isAllowed $=\mathrm{y}$

Villalón, X. G. (2014). El liderazgo transformacional como agente motivador en un establecimiento municipal [Tesis doctoral, Universidad de Chile]. http://repositorio.uchile.cl/bitstream/handle/2250/133625/TESIS \%20MAGISTER.pdf? $\underline{\text { sequence }=1 \& \text { is Allowed }=\mathrm{y}}$

Zeña, B. M. (2017). Gestión del potencial humano y liderazgo pedagógico de la Institución Educativa Nuestra Señora de Montserrat, Lima, 2016 [Tesis de maestría, Universidad César Vallejo]. https://repositorio.ucv.edu.pe/bitstream/handle/20.500.12692/8367/Ze\%C3\%B1a_CBM .pdf? sequence $=1$ 